\title{
Préparer les pharmaciens de demain : les pharmaciens d'établissements devraient-ils tous être prêts et aptes à former les étudiants en pharmacie?
}

\author{
par Lalitha Raman-Wilms
}

$\mathrm{L}$ 'une des valeurs de la Société canadienne des pharmaciens d'hôpitaux est " l'engagement des membres envers notre Société et la profession ${ }^{1}$. Cet engagement envers la profession comporte-t-il l'attente que les pharmaciens d'établissements deviennent en partie responsables de la formation des futurs praticiens? Adopter cette idée, ce qu'ont fait bon nombre de professionnels de la santé, fera en sorte que les fournisseurs de soins de demain seront bien formés.

Les programmes éducatifs en pharmacie au Canada se transforment en études de doctorat de premier cycle en pharmacie qui comprennent un enseignement axé davantage sur une formation expérientielle approfondie que ne l'étaient les programmes précédents de baccalauréat. Appeler tous les pharmaciens d'établissements à agir comme précepteurs pourrait aider les étudiants à acquérir une expérience concrète dans différents milieux, sous l'égide de praticiens chevronnés.

Dans le présent numéro, Cameron et collab. ${ }^{2}$ font part des stratégies employées par un hôpital universitaire à sites multiples, au sein d'un centre de santé universitaire, dont le but est d'accroître les possibilités de stages expérientiels pour les étudiants en pharmacie. L'une de leurs approches repose sur l'élaboration d'un " plan de gestion du changement pour l'ensemble de la pharmacie » qui établit « une charge d'enseignement minimum obligatoire pour tous les pharmaciens ". De plus, ils ont ajouté ce rôle de formateur aux évaluations annuelles du rendement des pharmaciens, ce qui indique que l'enseignement revêt de l'importance au même titre que les soins aux patients et les activités administratives.

Les centres de santé universitaires détiennent un mandat tripartite : prestation de soins, recherche et enseignement. L'accent est d'ailleurs mis sur la formation de la prochaine génération de

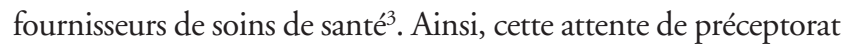

pourrait s'avérer plus réaliste envers les praticiens qui exercent dans le cadre d'un modèle de centre de santé universitaire. Mais qu'en est-il des pharmaciens travaillant dans les hôpitaux communautaires ou les plus petits établissements? Est-il raisonnable de s'attendre à ce que tous les pharmaciens exerçant en établissements (peu importe le type d'établissement) contribuent à la formation des étudiants? Travailler à inclure véritablement les étudiants au sein de la pratique quotidienne d'un pharmacien peut être avantageux pour les deux et peut aussi contribuer à améliorer les résultats thérapeutiques, même dans les petits établissements.

En 2011, la Cleveland Clinic Florida, un hôpital universitaire communautaire de 155 lits, a mis en œuvre un changement dans son modèle de pratique de la pharmacie dans le but de fournir des services de pharmacie à tous les patients hospitalisés ${ }^{4}$. Dans ce changement de modèle, ils ont inclus le modèle de pratique avec apprentissage hiérarchique [traduction libre de : Layered Learning Practice Model) $]^{5}$ afin de former les résidents et les étudiants en pharmacie. En intégrant réellement les apprenants dans leur pratique, les pharmaciens ont amélioré les soins qu'ils prodiguent. Ils ont aussi été en mesure de soutenir véritablement les facultés de pharmacie en augmentant le nombre total de stages avancés en pharmacie (Advanced Pharmacy Practice Experience), passant de 98 à 226 en deux ans.

De leur côté, Cameron et collab. ${ }^{2}$ indiquent que les changements en profondeur opérés dans leur service de pharmacie leur ont permis d'atteindre "sur quatre ans un volume de stages 3,5 fois plus important».

En ce qui a trait à l'influence sur les soins aux patients, le changement de modèle de pratique à la Cleveland Clinic Florida ${ }^{4}$ était associé à un accroissement du taux de satisfaction des patients (passant de $58 \%$ à $70 \%$ en seulement 18 mois) et à un effet positif dans le domaine de la « communication de médicaments ». 
De plus, le nombre d'interventions pharmaceutiques par jourpatient a augmenté, passant de 0,9 à 1,4 . La présence de stagiaires en pharmacie a aussi aidé l'établissement à respecter ses exigences en ce qui touche au bilan comparatif des médicaments. La non-disponibilité des étudiants durant certains mois et les difficultés à assurer la permanence les soirs et les fins de semaine ont représenté des obstacles qui ont nécessité des discussions continues avec les facultés de pharmacie quant à la structure des stages et aux heures de service.

Selon un article récent de la rubrique Director's Forum dans la revue Hospital Pharmacy, Weber ${ }^{6}$ faisait remarquer que l'un des principaux enjeux des leaders de la pharmacie aux États-Unis en 2015 concernait la Pharmacy Practice Model Initiative (renommée dernièrement Practice Advancement Initiative) lancée par l'American Society of Health-System Pharmacists (ASHP)7 conformément à un énoncé de principe de l'ASHP qui a découlé de ce projet, il y soulignait un intérêt grandissant pour " continuer d'accroître le rôle des étudiants en pharmacie » [traduction libre] $]^{6}$. L'objectif du projet est d'étendre les services de pharmacie à l'aide d'approches qui accroittront le rôle du pharmacien « tout au long du continuum des soins " [traduction libre] ${ }^{6}$. Ce modèle de pratique reconnaît la valeur des étudiants en pharmacie dans son cadre en leur confiant des rôles intégrés et des responsabilités. En intégrant réellement les étudiants dans la pratique quotidienne, on arrive à améliorer les services des pharmaciens et l'effet sur les résultats thérapeutiques.

Alors qu'on affronte des compressions continuelles dans les établissements, la contrainte de devoir en faire plus avec moins ainsi que les attentes de résultats thérapeutiques positifs, les étudiants, agissant comme « rallonges » des pharmaciens, représentent une solution pour permettre à ces derniers ainsi qu'aux établissements d'atteindre leurs buts en ce qui concerne les soins aux patients. Les pharmaciens œuvrant au sein d'établissements petits ou grands, universitaires ou communautaires, peuvent améliorer les soins qu'ils prodiguent en incorporant de façon efficace les activités des apprenants dans leur pratique quotidienne. Les établissements et le milieu universitaire doivent donc collaborer étroitement pour élaborer des indicateurs de réussite qui contiennent des paramètres précis liés aux activités de soins aux patients. De plus, établir conjointement des facteurs qui permettent de déterminer si les étudiants sont prêts pour la pratique peut aider les universités à mieux évaluer leurs programmes. Mais pour que plus de pharmaciens endossent le rôle de formateur, des investissements destinés au soutien adéquat des milieux d'exercice et à la formation des précepteurs sont aussi essentiels.
En entretenant un partenariat de collaboration entre les établissements et les facultés ou les écoles de pharmacie, nous nous assurons que le patient, le pharmacien, l'étudiant, l'établissement universitaire et le système de santé en profiteront tous! Mais une telle collaboration exige des pharmaciens et des établissements qu'ils adoptent le rôle de praticien-enseignant et elle nécessite des établissements universitaires et des milieux cliniques qu'ils travaillent ensemble dans l'intérêt mutuel du patient et de la profession.

[Traduction par l'éditeur]

\section{Références}

1. About us: CSHP's values. Ottawa $(\mathrm{ON})$ : Société canadienne des pharmaciens d'hôpitaux; 2015. Publié au : www.cshp.ca/aboutUs/ourMission_e.asp. Consulté le 4 décembre 2015.

2. Cameron K, Fernandes O, Musing ELS, Raymond C. Increasing capacity for experiential rotations for pharmacy learners: lessons learned from a multisite teaching hospital. Can J Hosp Pharm. 2016;69(1):23-9.

3. Trois missions un seul avenir : optimiser le rendement des Centres des sciences de la santé universitaires du Canada. Groupe de travail national sur l'avenir des Centres des sciences de la santé universitaires du Canada; 2010. Publié au : http://www.healthcarecan.ca/wp-content/uploads/2014/11/05.30-NTFFR-FINAL-V2.pdf. Consulté le 4 décembre 2015.

4. Delgado O, Kernan WP, Knoer SJ. Advancing the pharmacy practice model in a community teaching hospital by expanding student rotations. Am J Health Syst Pharm. 2014;71(1):1871-6.

5. Pinelli NR. The Layered Learning Practice Model: toward a consistent model of pharmacy practice [résumé]. Bethesda (MD) : ASHP Foundation. Publié au : www.ashpfoundation.org/PinelliAbstract. Consulté le 4 décembre 2015.

6. Weber RJ. Directors' Forum: issues facing pharmacy leaders in 2015: suggestions for pharmacy strategic planning. Hosp Pharm. 2015;50(2):167-72.

7. Pharmacy Practice Model Initiative: overview. Bethesda (MD) : American Society of Health-System Pharmacists. Publié au : www.ashpmedia.org/ppmi/ overview.html. Consulté le 4 décembre 2015.

Lalitha Raman-Wilms, B. Sc. (Phm.), Pharm. D., FCSHP, est vice-doyenne, Enseignement, et professeure agrégée à la Faculté de pharmacie Leslie Dan de I'Université de Toronto à Toronto, en Ontario. Elle est également rédactrice adjointe du $\mathrm{JCPH}$.

Intérêts concurrents : Lalitha Raman-Wilms supervise l'administration de la formation expérientielle des étudiants à la Faculté de pharmacie Leslie Dan de l'Université de Toronto (y compris les stages se déroulant au centre de santé universitaire dont il est question dans I'article de Cameron et collab. ${ }^{2}$ ). L'auteure n'a déclaré aucun autre intérêt concurrent.

\section{Adresse de correspondance :}

Dre Lalitha Raman-Wilms

Faculté de pharmacie Leslie Dan

Université de Toronto

144, rue College

Toronto (ON) M5S 3M2

Courriel : I.raman.wilms.a@utoronto.ca 